\title{
Analysis and Testing of MR Shear Transmission Driven by SMA Spring
}

\author{
Jin Huang, ${ }^{1}$ Xu Chen, ${ }^{2}$ and Lirong Zhong ${ }^{1}$ \\ ${ }^{1}$ College of Mechanical Engineering, Chongqing University of Technology, Chongqing 400050, China \\ ${ }^{2}$ College of Vehicle Engineering, Chongqing University of Technology, Chongqing 400050, China \\ Correspondence should be addressed to Xu Chen; chenxu12@cqut.edu.cn
}

Received 11 July 2013; Accepted 27 August 2013

Academic Editor: Xing Chen

Copyright (c) 2013 Jin Huang et al. This is an open access article distributed under the Creative Commons Attribution License, which permits unrestricted use, distribution, and reproduction in any medium, provided the original work is properly cited.

\begin{abstract}
A transmission method of magnetorheological (MR) fluid in cylindrical-type driven by shape memory alloy (SMA) spring with the thermal effect was proposed. The torque can change rapidly with temperature. Based on the thermal effect of SMA, the expression for displacement of SMA spring was established. The rheological property of MR fluid under the magnetic fields was described by Bingham model. Based on momentum equation, the shear flow of MR fluid between two cylinders was analyzed and the expressions of flow velocity and torque have been obtained. The experimental results show the output displacement of SMA spring and the transmit torque of MR fluid is directly proportional to the temperature and the applied magnetic field, respectively.
\end{abstract}

\section{Introduction}

MR fluids and SMAs are known as smart materials for their properties can change rapidly on different external conditions. By applying a magnetic field, the fluids show the viscoplastic behavior with yield strength $[1,2]$. Altering the strength of the magnetic field will precisely control the shear yield stress of the fluid. MR fluids are useful for the efficient control of the torques and forces transmission, and they can be used in clutches [3-7], brakes [8,9], dampers [10-12], valves $[13,14]$, and so on. SMAs may undergo mechanical shape changes at relatively low temperatures and retain them until heated and then coming back to the initial shape $[15,16]$. This makes SMAs unique compared to other smart materials that can be used for actuator applications $[17,18]$.

MR transmission is a device that transmits torque by the shear stress of the MR fluids. It has the property that their transmitting torque changes quickly in response to an external magnetic field. A literature review indicates that the transmission using MR fluids can be classified as having either disc-type or cylindrical-type [6]. In the disc-type, the MR fluid within the activation region between two parallel discs is a circular plate. In the cylindrical-type, the activated MR fluid between two concentric cylinders is an annular cylinder.
In order to rationally design the MR transmission devices driven by SMA spring under thermal effect a designer should analyzes the output displacement of SMA springunder thermal effect, and the torque transmission ability of MR fluid. In this paper, Bingham model is used to describe the constitutive characteristics of MR fluids subject to an applied magnetic field. The expressions for the output displacement of SMA spring and the torque of the cylindrical type MR transmission are established to provide the theoretical foundation for the design of MR device.

\section{Transmission Principle}

The schematic of the cylindrical MR transmission driven by SMA spring under thermal effect is shown in Figure 1. The driving shaft and driving cylinder are initiative members, and the shell is the driven one. The initiative members rotate at definite velocity $\omega_{1}$. At low temperature, MR fluid is saved at oil chamber, and the transmission system is in the state of separation. When the temperature gets higher, the SMA driving spring will pushes the MR fluid filling into the working gap between the driving-cylinder and shell through pipe rapidly. The cylindrical MR transmission device depends on the shear stress of the MR fluid to transmit torque. In the absence of 


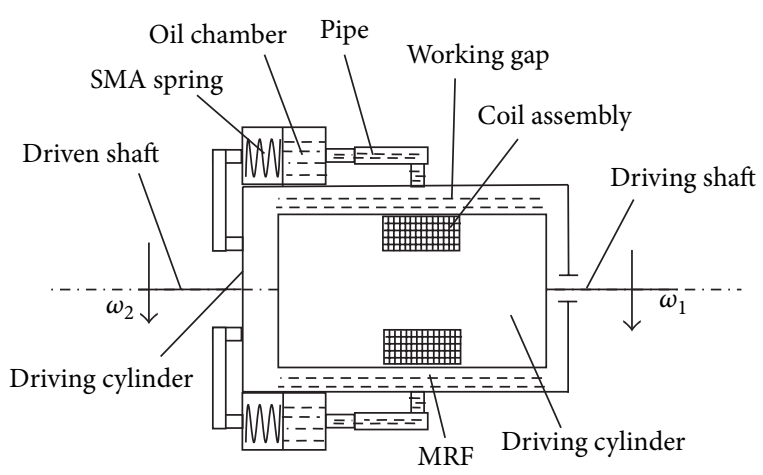

FIGURE 1: MR fluid shear transmission driven by SMA between two cylinders.

an applied magnetic field, MR fluid keeps flowing, so its transmission torque is only a very small viscous torque. However, in the course of operation, a magnetic flux path is formed when electric current is put through the coil assembly. As a result, the MR particles are gathered to form chain-like structures, in the direction of the magnetic flux path. These chainlike structures increase the shear stress of the MR fluid. With the increase of the applied magnetic field, the shear stress developed by the MR fluid goes up rapidly. When the shear stress is large enough, the initiative and driven members can finish a synchronous rotation. The amount of torque can be adjusted continuously by changing the input current in coil assembly.

\section{Thermal Characteristics of SMA Spring}

The displacement of bias type SMA actuating device based on SMA spring can be expressed as follows [19]:

$$
S(T)=\frac{\left(G(T)-G_{\mathrm{M}}\right) \Delta \delta F_{\mathrm{L}} \gamma_{1 \mathrm{~L}}}{\left(d / n \pi D^{2}\right) G(T) \Delta \delta F_{\mathrm{L}}+\left(F_{\mathrm{H}}-F_{\mathrm{L}}\right) G_{\mathrm{M}} \gamma_{1 \mathrm{~L}}},
$$

where $S(T)$ is the displacement of temperature control switch at temperature $T, d$ is the diameter of SMA wire, $D$ is the diameter of spring, $n$ is the number of spring turns, $G_{M}$ is the elasticity modulus of martensite phase, $\gamma_{1 \mathrm{~L}}$ is the shear strain of martensite at low temperature, $F_{\mathrm{L}}$ and $F_{\mathrm{H}}$ are axial loads of SMA spring in martensite phase and austenitic phase, respectively, $\Delta \delta$ is the maximum displacement of bias type SMA temperature controlled switch, and $G(T)$ is the shear elasticity modulus of SMA spring at temperature $T$.

Consider

$$
\begin{aligned}
& G(T) \\
& = \begin{cases}G_{\mathrm{M}} & T<M_{\mathrm{f}}, T<A_{\mathrm{s}} \\
G_{\mathrm{M}}+\frac{G_{\mathrm{A}}-G_{\mathrm{M}}}{2}\left[1+\sin \varphi\left(T-T_{m}\right)\right] & M_{\mathrm{f}} \leq T \leq A_{\mathrm{f}} \\
G_{\mathrm{A}} & T>A_{\mathrm{f}}, T>M_{\mathrm{s}},\end{cases}
\end{aligned}
$$

where $G_{\mathrm{A}}$ is the elasticity modulus of austenitic phase and $M_{\mathrm{s}}$ and $A_{\mathrm{s}}$ are the starting values of phase change temperature for martensite and austenitic, respectively. $M_{\mathrm{f}}$ and $A_{\mathrm{f}}$ are the final values of phase change temperature, respectively. In the heating process, $T_{m}=\left(A_{\mathrm{s}}+A_{\mathrm{f}}\right) / 2$ and $\varphi=\pi /\left(A_{\mathrm{f}}-A_{\mathrm{s}}\right)$.

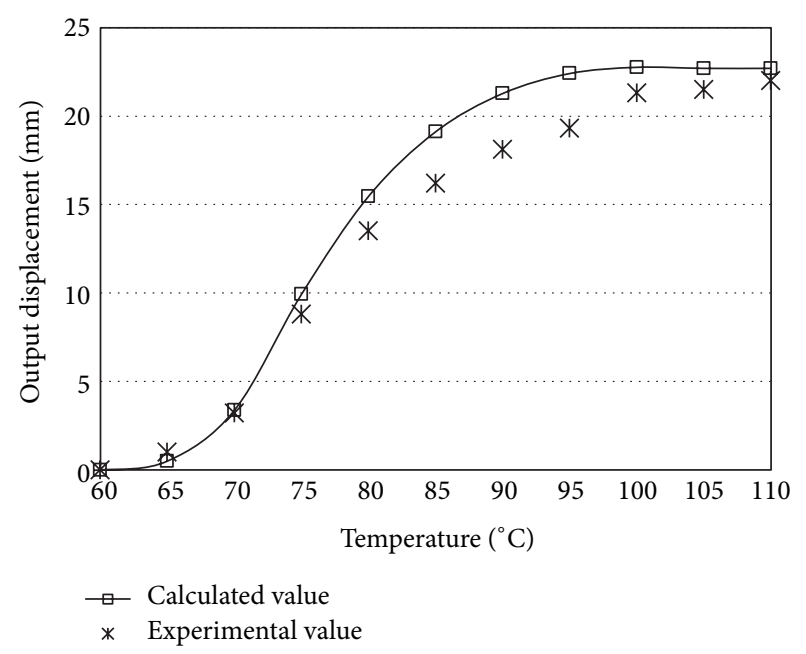

FIGURE 2: The displacements of SMA spring versus temperatures.

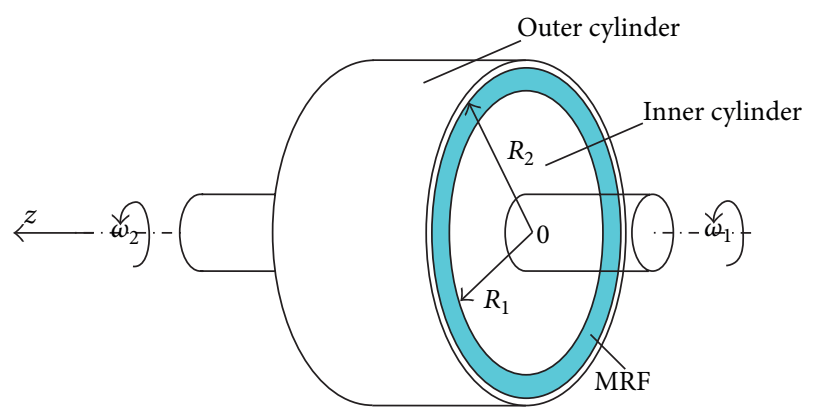

FIgURE 3: Circular flow of MR fluid between two cylinders.

In the cooling process, $T_{m}=\left(M_{\mathrm{f}}+M_{\mathrm{s}}\right) / 2$ and $\varphi=\pi /\left(M_{\mathrm{s}}-\right.$ $M_{\mathrm{f}}$ ).

The theoretical and the experimental results of the relationship between output displacements and temperatures of SMA spring are shown in Figure 2. The SMA is NiTi alloy, and the phase transition temperatures are $M_{\mathrm{f}}=50^{\circ} \mathrm{C}, M_{\mathrm{s}}=$ $78^{\circ} \mathrm{C}, A_{\mathrm{s}}=74^{\circ} \mathrm{C}$, and $A_{\mathrm{f}}=95^{\circ} \mathrm{C}$. The shear elasticity modulus at austenitic and martensite phase is $G_{\mathrm{A}}=25 \mathrm{GP}$ and $G_{\mathrm{M}}=7.5 \mathrm{GPa}$, respectively; the shear strain of martensite at low temperature is $\gamma_{1 \mathrm{~L}}=2 \%$. The parameters of spring are as follows: $D=8.5 \mathrm{~mm}, d=1 \mathrm{~mm}, n=7$, and the angle of inclination $\alpha=6^{\circ}$.

\section{The Shear Transmission of MR Fluid between Two Cylinders}

Figure 3 shows the circular flow of an MR fluid within the operational gap between two cylinders. When the inner cylinder is rotated by the rotating speed $\omega_{1}$, the MR fluid is sheared and flowed at the rotating speed $\omega_{r}$ in $\theta$ direction. The outer cylinder is rotated by the rotating speed $\omega_{2}$ because of the shear stress of the fluid. 
In the cylindrical coordinate system, the momentum equation of the shear flow in $\theta$ direction can be expressed by

$$
\begin{aligned}
& \rho\left(\frac{\partial v_{\theta}}{\partial t}+\frac{v_{\theta}}{r} \frac{\partial v_{\theta}}{\partial \theta}+v_{z} \frac{\partial v_{\theta}}{\partial z}\right) \\
& =f_{\Theta}-\frac{1}{r} \frac{\partial p}{\partial \theta}+\frac{\partial \tau_{r \theta}}{\partial r}+\frac{1}{r} \frac{\partial \tau_{\theta \theta}}{\partial \theta}+\frac{\partial \tau_{z \theta}}{\partial z}+\frac{2}{r} \tau_{r \theta},
\end{aligned}
$$

where $\rho$ is the density of MRF, $v_{\theta}$ is the flow velocity of the fluid in the $\theta$ direction, $t$ is time, $f_{\Theta}$ is the mass force in $\theta$ direction, and $\tau_{i j}$ is the shear stress.

In order to analyze the behavior of MR fluids flow in the gap between two cylinders, the following assumptions are given: the fluid is incompressible. There is no flow in radial direction and axial direction, but only tangential flow. The flow velocity of MR fluid is a function of radius. The pressure in the thickness direction of MR fluid is a constant one. The strength of magnetic field in the working gap of the activation region is well distributed. In the cylindrical coordinate system $(r, \theta, z), z$-direction is coincident with the axis of two cylinders, and the distribution of the flow velocity is

$$
v_{r}=0 \quad v_{\theta}=r \omega(r) \quad v_{z}=0,
$$

where $v_{r}$ and $v_{z}$ are the flow velocity of the fluid in the $r$ direction and $z$-direction, respectively. $\omega(r)$ is the rotation angular velocity of the fluid in the $\theta$ direction. The angular velocity $\omega(r)$ is the function of $r$-coordinate.

In the mode of flow, assume that the MR fluid within the gap is regarded as a cylindrical fluid. The momentum equation of the shear flow at the narrow gap may be approximated by

$$
\frac{\mathrm{d} \tau_{r \theta}}{\mathrm{d} r}+\frac{2}{r} \tau_{r \theta}=0,
$$

where $\tau_{r \theta}$ is the shear stress developed by MR fluid.

Ignore the wall slip effect, and the boundary conditions of MR fluid flow can be expressed as

$$
\begin{aligned}
& \left.v_{\theta}\right|_{r=R_{1}}=R_{1} \omega_{1}, \\
& \left.v_{\theta}\right|_{r=R_{2}}=R_{2} \omega_{2} .
\end{aligned}
$$

The behavior of MR fluids is similar to Newtonian fluid without applied magnetic field. When a magnetic field is applied, the characteristics of MR fluids can be described by Bingham model [20]:

$$
\begin{gathered}
\tau_{r \theta}=\tau_{y}+\eta \dot{\gamma} \quad \tau \geq \tau_{y}, \\
\dot{\gamma}=0 \quad \tau<\tau_{y} .
\end{gathered}
$$

Integrating the momentum equation (5), shear stress can be indicated as

$$
\tau_{r \theta}=\frac{c_{1}}{r^{2}}
$$

where $c_{1}$ is the integrating constant. Because the rotating speed decreases with the increase of the radius $r$, and the shear rate $\dot{\gamma}$ in (7) can be calculated by

$$
\dot{\gamma}_{r \theta}=-r \frac{\mathrm{d} \omega(r)}{\mathrm{d} r} .
$$

The flow velocity $\omega(r)$ can be obtained from (7), (8), and (9) as

$$
\omega(r)=\frac{\tau_{y}}{\eta} \ln r+\frac{c_{1}}{2 \eta r^{2}}+c_{2},
$$

where $c_{2}$ is the integrating constant.

Applying the boundary conditions in ((6a) and (6b)), the integrating constants can be determined as

$$
\begin{gathered}
c_{1}=\frac{2 R_{1}^{2} R_{2}^{2} \tau_{y}}{R_{2}^{2}-R_{1}^{2}} \ln \frac{R_{2}}{R_{1}}+\frac{2 \eta R_{1}^{2} R_{2}^{2}}{R_{2}^{2}-R_{1}^{2}}\left(\omega_{1}-\omega_{2}\right), \\
c_{2}=\frac{R_{2}^{2} \omega_{2}-R_{1}^{2} \omega_{1}}{R_{2}^{2}-R_{1}^{2}}-\frac{\tau_{y}}{\eta}\left(\frac{R_{2}^{2} \ln R_{2}}{R_{2}^{2}-R_{1}^{2}}-\frac{R_{1}^{2} \ln R_{1}}{R_{2}^{2}-R_{1}^{2}}\right) .
\end{gathered}
$$

Submit ((11a) and (11b)) into (10), and the velocity distribution can be obtained as

$$
\begin{aligned}
\omega(r)= & \frac{\tau_{y}}{\eta} \ln r+\frac{R_{1}^{2} R_{2}^{2}}{R_{2}^{2}-R_{1}^{2}}\left(\frac{\tau_{y}}{\eta} \ln \frac{R_{2}}{R_{1}}+\omega_{1}-\omega_{2}\right) \frac{1}{r^{2}} \\
& +\frac{R_{2}^{2} \omega_{2}-R_{1}^{2} \omega_{1}}{R_{2}^{2}-R_{1}^{2}}-\frac{\tau_{y}}{\eta}\left(\frac{R_{2}^{2} \ln R_{2}}{R_{2}^{2}-R_{1}^{2}}-\frac{R_{1}^{2} \ln R_{1}}{R_{2}^{2}-R_{1}^{2}}\right) .
\end{aligned}
$$

As shown in Figure 3, MR fluids flow between two cylinders. The transmission torque of MR fluid can be expressed as

$$
M=2 \pi r^{2} L \tau,
$$

where $L$ is the width of the working gap. There are two parts of transmission torque of MR fluids between two cylinders, which are torque $M_{\eta}$ produced by viscosity of MR fluids and torque $M_{H}$ produced by yield stress $\tau_{y}$. If all of the MR fluids in working gap between two cylinders are yielded in shear mode, the transmission torque $M_{\eta}$ produced by viscosity of MR fluids can be derived by combining (7), (9), (12), and (13):

$$
M_{\eta}=4 \pi \eta L \frac{\left(\omega_{1}-\omega_{2}\right) R_{1}^{2} R_{2}^{2}}{R_{2}^{2}-R_{1}^{2}} .
$$

The torque $M_{H}$ produced by yield stress of MR effect can be described as

$$
M_{H}=\frac{\pi L_{e}\left(R_{2}+R_{1}\right)^{2}}{2} \tau_{y}
$$

where $L_{e}$ is the effective width of the MR effect developed by the MR fluid. The transmission torque of MR fluids between two cylinders is

$$
M=M_{H}+M_{\eta}=\frac{\pi L_{e}\left(R_{2}+R_{1}\right)^{2}}{2} \tau_{y}+4 \pi \eta L \frac{\left(\omega_{1}-\omega_{2}\right) R_{1}^{2} R_{2}^{2}}{R_{2}^{2}-R_{1}^{2}} .
$$




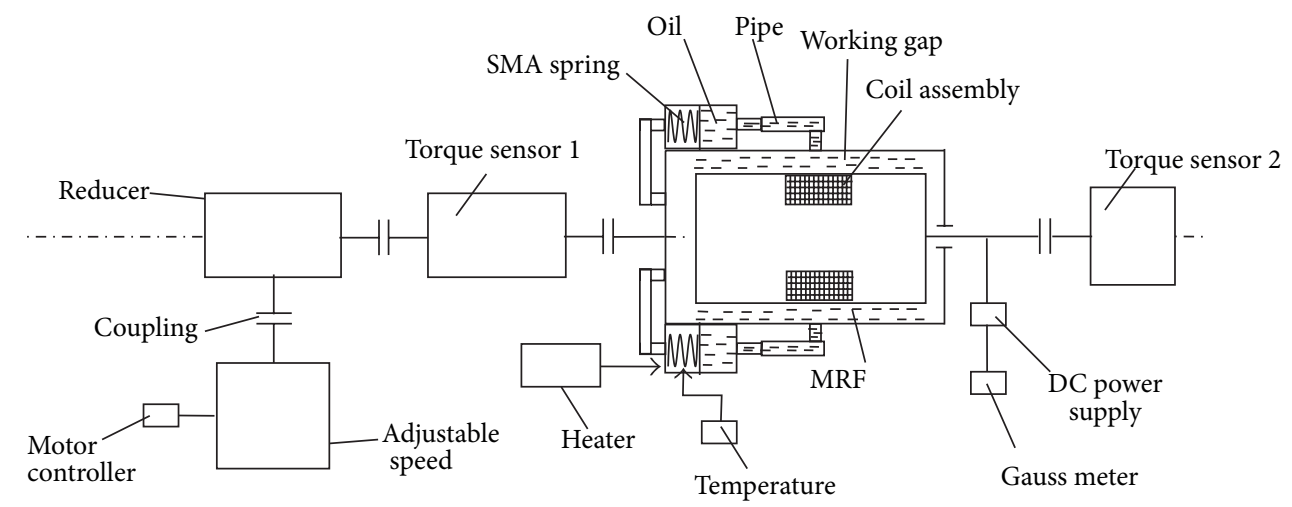

FIGURE 4: The overall sketch of performance experimental system for cylindrical-type MR fluid transmission driven by shape memory alloy.

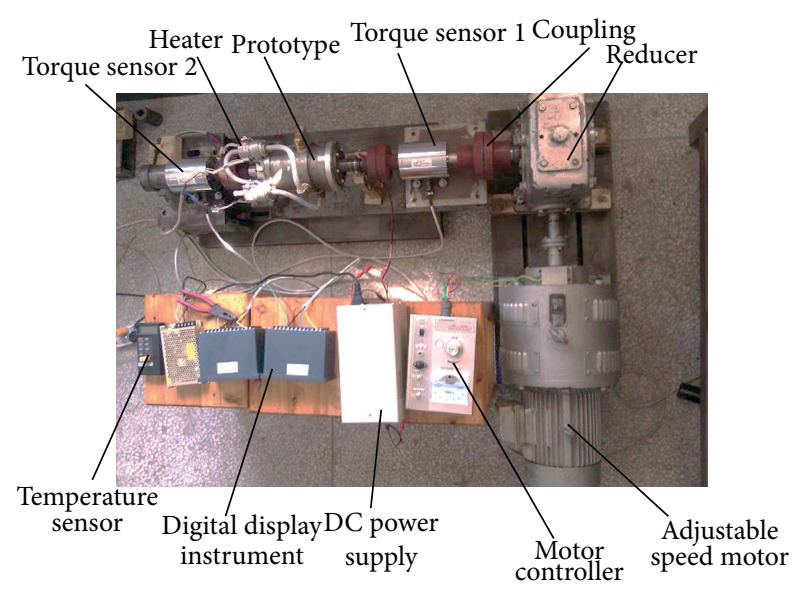

Figure 5: The performance experimental system for cylindricaltype MR fluid transmission driven by shape memory alloy.

\section{Test and Analysis}

Figure 4 shows the overall sketch of performance experimental system for cylindrical-type MR fluid transmission driven by shape memory alloy. Based on this system, the transmission torques of MR fluids between two cylinders under different applied magnetic fields are analyzed. The shearing rate of MR fluids between two cylinders can be adjusted by motor in the test system; see Figure 5. The magnetic field strength can be controlled by electric current in coil. All parameters in system are measured in real time by temperature sensor, speed, and torque sensors.

For this example, we use a typical MR fluid. Figure 6 shows the relation, obtained from the experiment, between the dynamic yield stress and the magnetic field strength for a typical MR fluid. The result shows that the shear stress is heavily influenced by applied magnetic field. In this study, the following parameters are given: $\eta=0.86 \mathrm{~Pa} \cdot \mathrm{s}, L=140 \mathrm{~mm}$, $L_{e}=50 \mathrm{~mm}, R_{1}=50$, and $R_{2}=52$.

When temperature is $60^{\circ} \mathrm{C}$ and the speed of driving shaft is $60 \mathrm{r} / \mathrm{min}$, the relationship between the transmission torque of MR fluid between two parallel cylinders and the field current in coil assembly was obtained according to the test

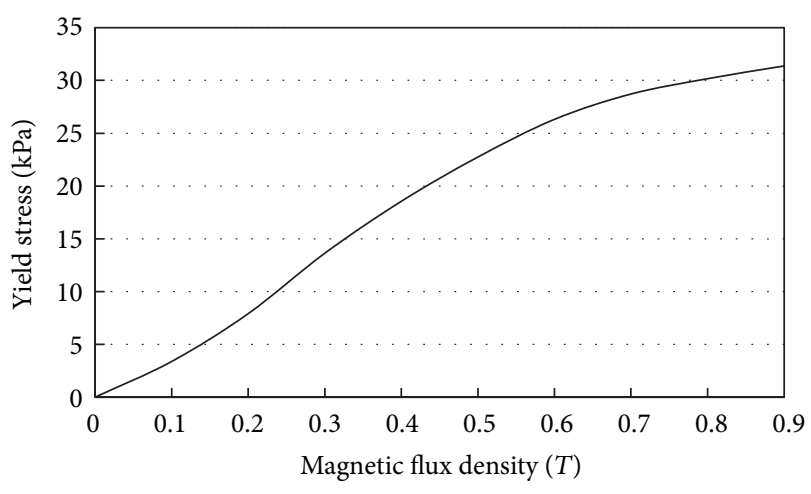

FIGURE 6: Yield stress versus magnetic flux density.

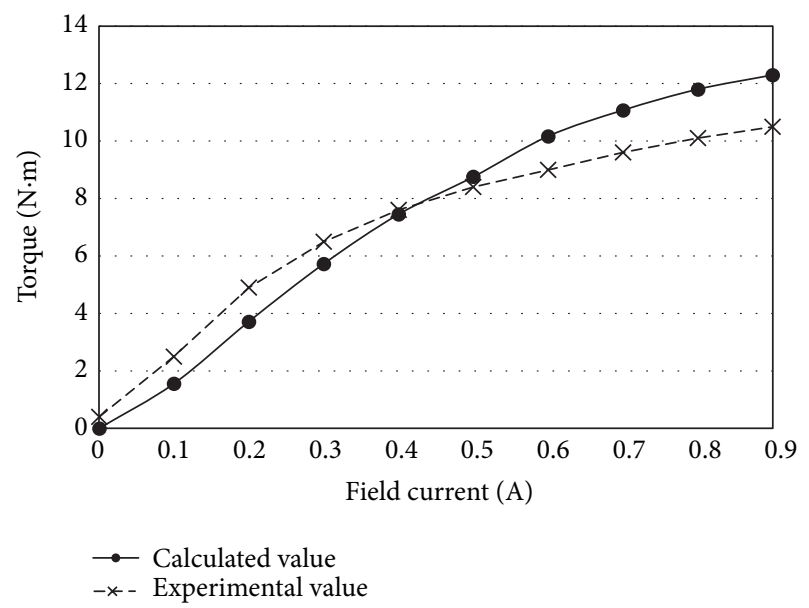

Figure 7: Torque versus field current.

system in Figure 5. The comparison of the theoretical and the experimental results is shown in Figure 7. When the currents are $0 \mathrm{~A}, 0.2 \mathrm{~A}, 0.4 \mathrm{~A}, 0.6 \mathrm{~A}$, and $0.8 \mathrm{~A}$, the experimental results of transmit torque are $0.6 \mathrm{~N} \cdot \mathrm{m}, 4.9 \mathrm{~N} \cdot \mathrm{m}, 7.6 \mathrm{~N} \cdot \mathrm{m}, 9.0 \mathrm{~N} \cdot \mathrm{m}$, and $10.1 \mathrm{~N} \cdot \mathrm{m}$, respectively. The transmit torque developed by MR fluid goes up rapidly with the increase of the applied magnetic field. The transmit torque with a variation of shear rate under field currents in coil assembly is shown in Figure 8. 


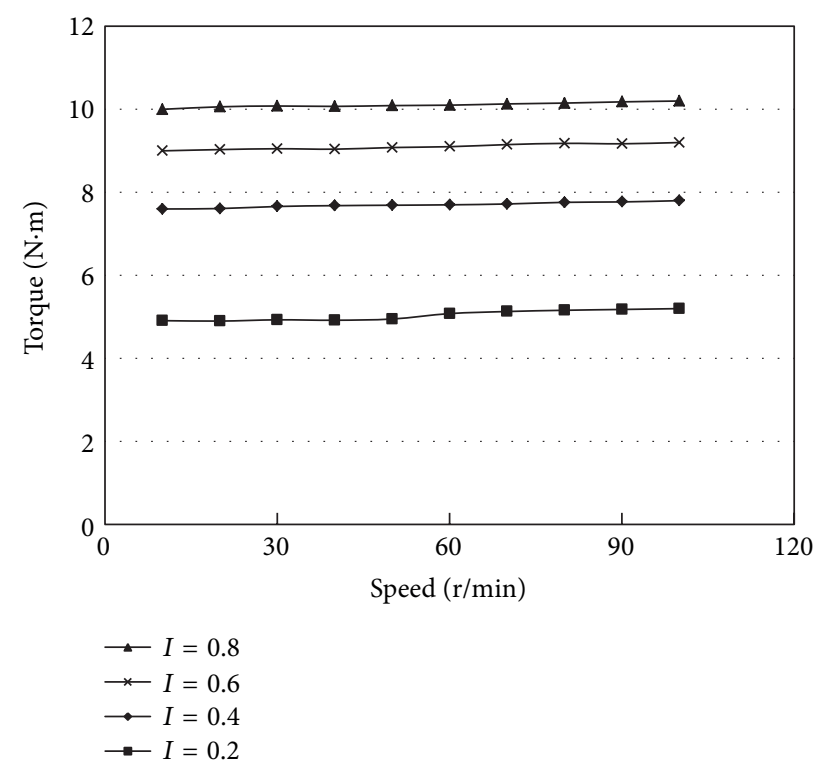

FIgURE 8: Torque versus speed.

In a variety of input speeds, the transmit torque remains generally stable.

The results show that the torque measured by experiment has the same trend with the calculated value basically. The deviation between experimental and theoretical values is brought by the error of yield stress, the structural error, the magnetic flux leakage, the simplification of theoretical analysis model, and so on.

\section{Conclusions}

(1) Based on the heating and driving characteristics of SMA, the relationship between displacement and temperature of SMA spring was analyzed. The experimental results were well satisfied with the theoretical results. In the range of phase transition temperature, the output displacement of SMA spring increases with the rising of temperature.

(2) The MR fluid can be driven into working chamber by SMA Spring. The volume of MR fluid in working chamber increases with the increasing of output displacement of SMA spring.

(3) Based on rheological characteristics of MR fluid, the flow and transmission of MR fluid between two cylinders were analyzed, and the equations of flow velocity and transmit torque of MR fluid between two cylinders were obtained. The transmit torque of MR fluid between two cylinders increases with the increasing of applied magnetic field.

\section{Acknowledgments}

This work was supported by the National Natural Science Foundation of China (51175532 and 11272368) and by the Natural Science Foundation Project of CQ CSTC (key project CSTC, 2011BA4028).

\section{References}

[1] M. S. Kim, Y. D. Liu, B. J. Park, C.-Y. You, and H. J. Choi, “Carbonyl iron particles dispersed in a polymer solution and their rheological characteristics under applied magnetic field," Journal of Industrial and Engineering Chemistry, vol. 18, no. 2, pp. 664-667, 2012.

[2] J. Zhang, J.-Q. Zhang, and J.-F. Jia, "Characteristic analysis of magnetorheological fluid based on different carriers," Journal of Central South University of Technology, vol. 15, no. 1, pp. 252-255, 2008.

[3] A. S. Shafer and M. R. Kermani, "On the feasibility and suitability of mr fluid clutches in human-friendly manipulators," IEEE/ASME Transactions on Mechatronics, vol. 16, no. 6, pp. 1073-1082, 2011.

[4] T. Kikuchi, K. Otsuki, J. Furusho et al., "Development of a compact magnetorheological fluid clutch for human-friendly actuator," Advanced Robotics, vol. 25, no. 9-10, pp. 1363-1363, 2011.

[5] P. Kielan, P. Kowol, and Z. Pilch, "Conception of the electronic controlled magnetorheological clutch," Przeglad Elektrotechniczny, vol. 87, no. 3, pp. 93-95, 2011.

[6] J. Huang, L. Fu, and L. Zhong, "Analysis of a magnetorheological transmission for fan clutch," Advanced Materials Research, vol. 287-290, pp. 173-177, 2011.

[7] Z. Herold, D. Libl, and J. Deur, "Design and testing of an experimental magnetorheological fluid clutch," Strojarstvo, vol. 52, no. 6, pp. 601-614, 2010.

[8] J. Huang, J. Q. Zhang, Y. Yang, and Y. Q. Wei, "Analysis and design of a cylindrical magneto-rheological fluid brake," Journal of Materials Processing Technology, vol. 129, no. 1-3, pp. 559-562, 2002.

[9] A. Farjoud, N. Vahdati, and Y. F. Fah, "Mathematical model of drum-type MR brakes using herschel-bulkley shear model," Journal of Intelligent Material Systems and Structures, vol. 19, no. 5, pp. 565-572, 2008.

[10] J. Huang, J. He, and G. Lu, "Analysis and design of magnetorheological damper," Advanced Materials Research, vol. 148-149, pp. 882-886, 2011.

[11] X. C. Zhu, X. J. Jing, and L. Cheng, "Magnetorheological fluid dampers: a review on structure design and analysis," Journal of Intelligent Material Systems and Structures, vol. 23, no. 8, pp. 839-873, 2012.

[12] E. Dragašius, V. Grigas, D. Mažeika, and A. Šulginas, "Evaluation of the resistance force of magnetorheological fluid damper," Journal of Vibroengineering, vol. 14, no. 1, pp. 1-6, 2012.

[13] M. Y. Salloom and Z. Samad, "Design and modeling magnetorheological directional control valve," Journal of Intelligent Material Systems and Structures, vol. 23, no. 2, pp. 155-167, 2012.

[14] J. Huang, J. M. He, and J. Q. Zhang, "Viscoplastic flow of the MR fluid in a cylindrical valve," Key Engineering Materials, vol. 274276, no. 1, pp. 969-974, 2004.

[15] G. Costanza, M. E. Tata, and C. Calisti, "Nitinol one-way shape memory springs: thermomechanical characterization and actuator design," Sensors and Actuators A, vol. 157, no. 1, pp. 113-117, 2010.

[16] S. Huang, M. Leary, T. Ataalla, K. Probst, and A. Subic, "Optimisation of Ni-Ti shape memory alloy response time by transient heat transfer analysis," Materials and Design, vol. 35, pp. 655$663,2012$. 
[17] S. Langbein and A. Czechowicz, "Adaptive resetting of SMA actuators," Journal of Intelligent Material Systems and Structures, vol. 23, no. 2, pp. 127-134, 2012.

[18] S. Degeratu, P. Rotaru, G. Manolea, H. O. Manolea, and A. Rotaru, "Thermal characteristics of Ni-Ti SMA (shape memory alloy) actuators," Journal of Thermal Analysis and Calorimetry, vol. 97, no. 2, pp. 695-700, 2009.

[19] J. Ma, H. Shu, and J. Huang, "MR continuously variable transmission driven by SMA for centrifugal fan in nuclear power plant," Science and Technology of Nuclear Installations, vol. 2012, Article ID 205675, 6 pages, 2012.

[20] J. D. Carlson and M. R. Jolly, "MR fluid, foam and elastomer devices," Mechatronics, vol. 10, no. 4, pp. 555-569, 2000. 

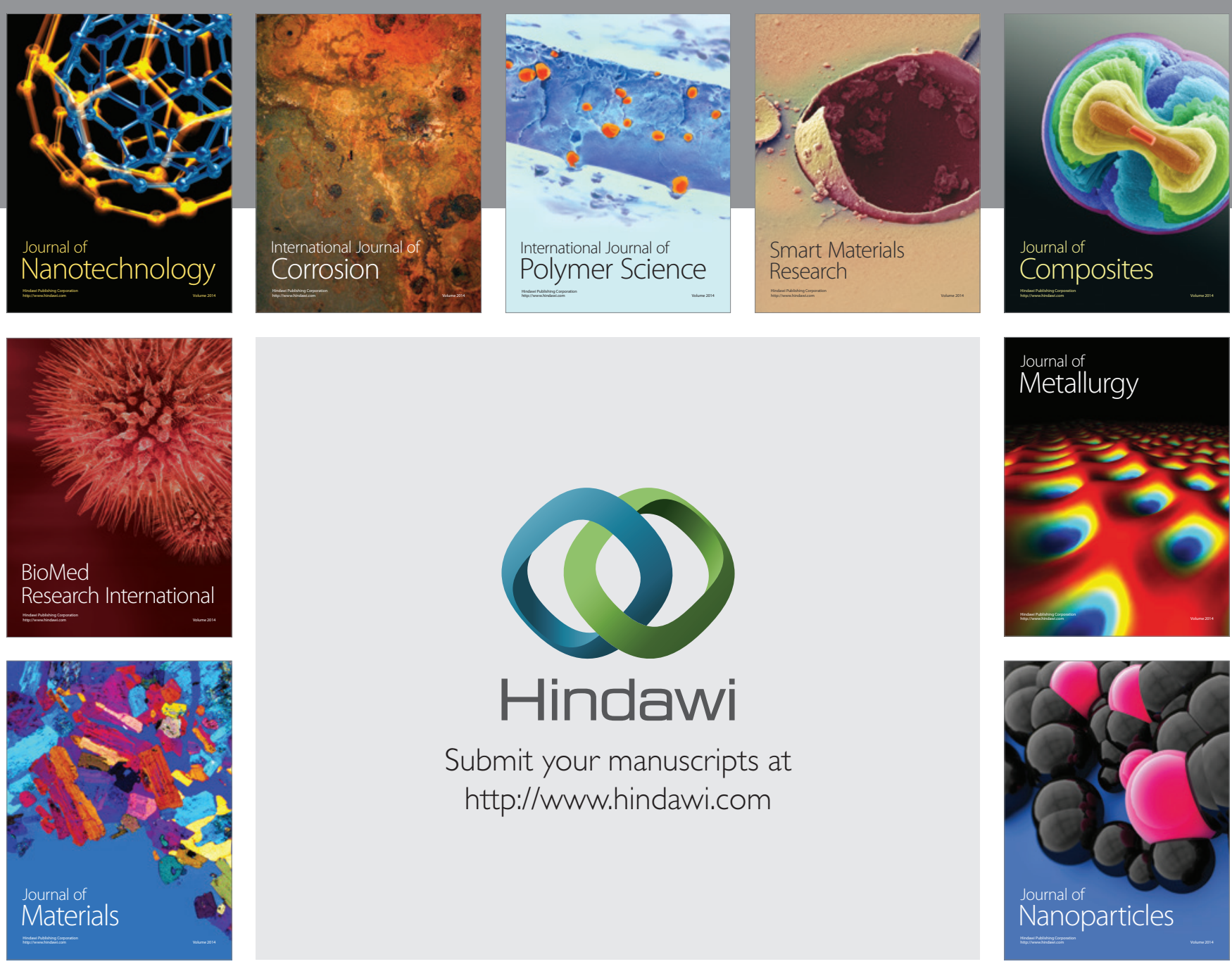

Submit your manuscripts at http://www.hindawi.com
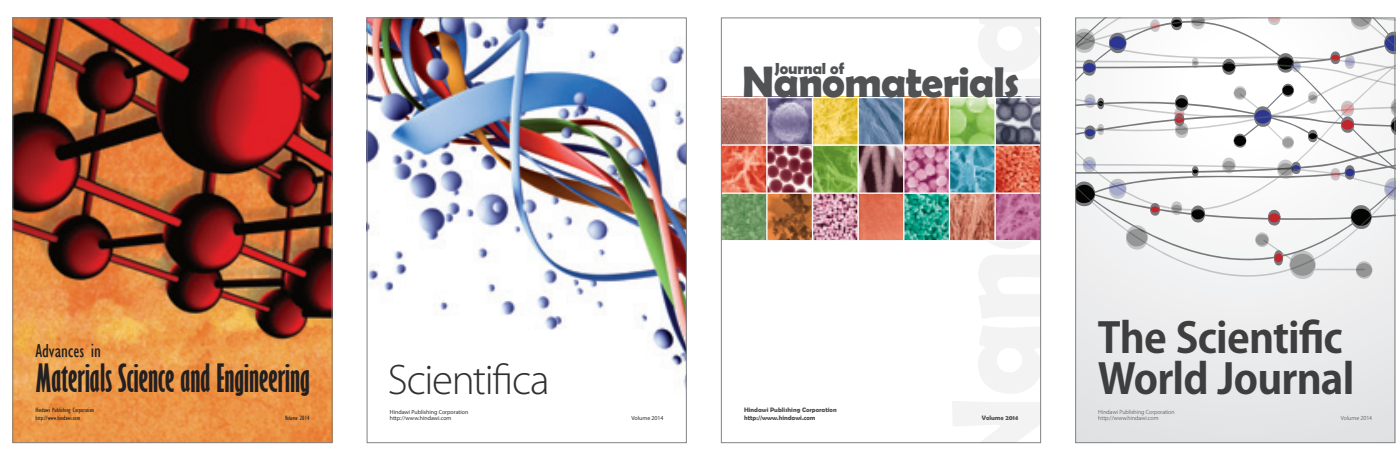

\section{The Scientific World Journal}
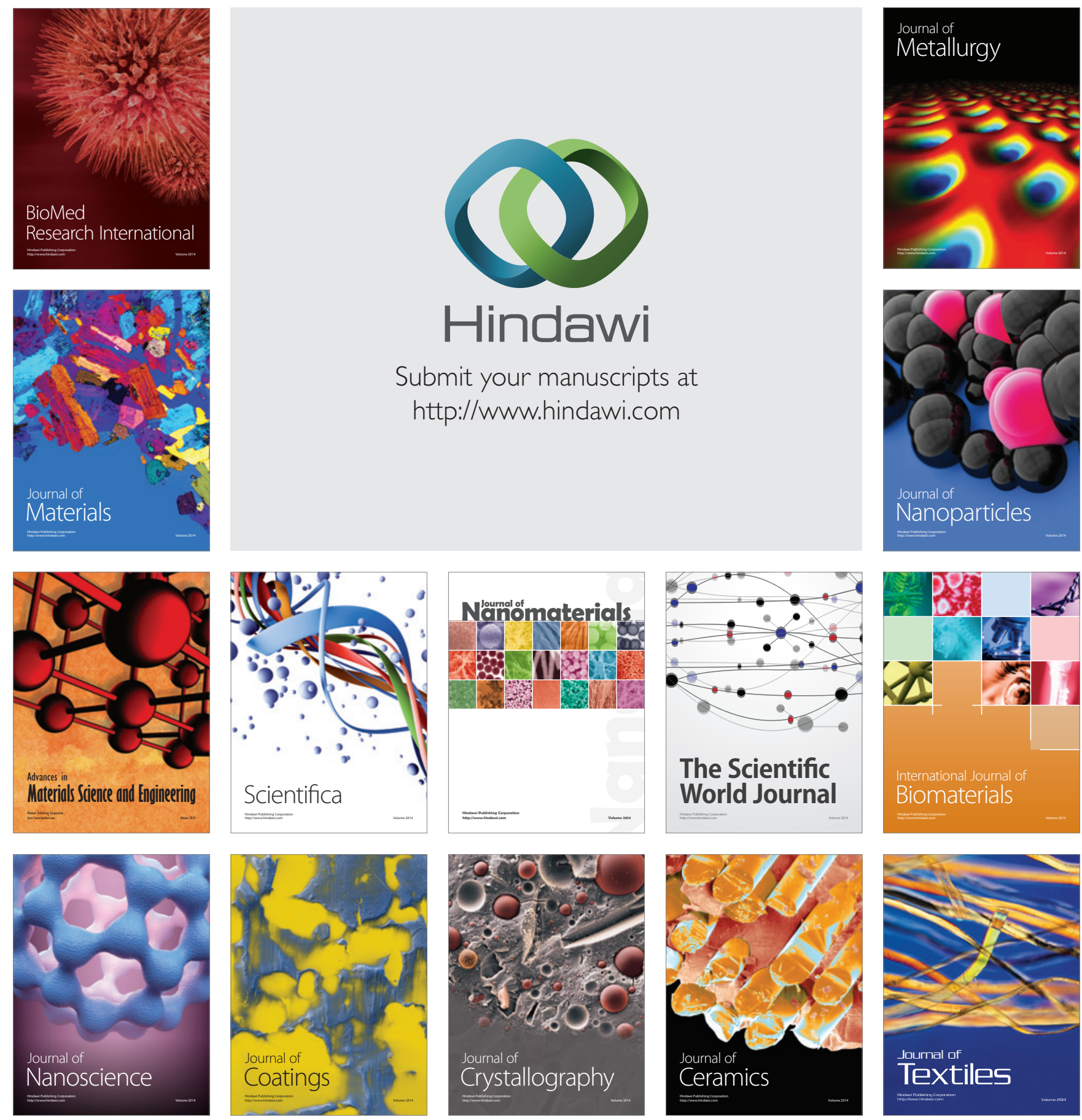